\title{
A pandemia da COVID-19 como questão sociocientífica: aportes do Instituto NUTES para professores e estudantes da educação básica
}

RESUMO

Isabel Gomes Rodrigues

\section{Martins}

isabelgrmartins@gmail.com Universidade Federal do Rio de Janeiro, Rio de Janeiro, Rio de Janeiro.

\section{Marcelo Borges Rocha}

rochamarcelo36@yahoo.com.br Centro Federal de Educação Tecnológica Celso Suckow da Fonseca, Rio de Janeiro, Rio de Janeiro.

Maria Angélica Mejia-Caceres missangelux@hotmail.com Universidade Federal do Rio de Janeiro, Rio de Janeiro, Rio de Janeiro.

Pedro Miguel Marques da Costa pedro_mmco@hotmail.com Centro Federal de Educação Tecnológica Celso Suckow da Fonseca, Rio de Janeiro, Rio de Janeiro.

Sandra Lopes Machado sandra.ufrj@yahoo.com.br
Este trabalho trata a pandemia da COVID-19 na perspectiva da abordagem das questões sóciocientíficas. Para tanto, apresenta a criação de um site com potencial para abordar aspectos conceituais, históricos, econômicos e sócio-políticos, numa perspectiva contextualizada e interdisciplinar. Mobilizou um grupo de docentes e estudantes de graduação e pós-graduação que analisou controvérsias e fake news sobre a pandemia, definiu critérios para seleção de materiais, e propôs perguntas motivadoras e atividades educativas na produção de uma página web dirigida a professores de ciências da educação básica. O site esclarece aspectos relacionados à pandemia e dá suporte para abordagens curriculares do tema em tempos de isolamento social.

PALAVRAS-CHAVE: Questões sociocientíficas. COVID-19. Educação em Ciências. Controvérsia. 
Comprometido com a produção científica que visa à transformação de práticas e programas de formação em Educação em Ciências e Saúde, o Instituto NUTES de Educação em Ciências e Saúde da Universidade Federal do Rio de Janeiro conta com uma equipe multiprofissional que realiza trabalhos de natureza interdisciplinar. No contexto da pandemia de Covid19, estabeleceu quatro grupos de trabalho (GT) dos quais participam servidores, docentes e técnicoadministrativos, pesquisadores em diferentes etapas de formação - da graduação ao pós-doutorado - e pesquisadores associados ao Programa de Pós-graduação Educação em Ciências e Saúde. O GT de Comunicação tem como propósito divulgar eventos e compartilhar conteúdos sobre questões relacionadas à pandemia; o GT de Recursos Educacionais organiza um acervo on-line dos materiais educativos produzidos pelo NUTES, incluindo aqueles que tratam especificamente da COVID19; o GT de Apoio a Situações Especificas coordena ações de suporte material e emocional ao corpo social da unidade; e o GT Acadêmico explora as contribuições da pesquisa em Educação em Ciências e Saúde ao tratamento curricular da pandemia.

Nesta nota, apresentamos o trabalho realizado pelo GT Acadêmico. O grupo caracteriza a pandemia da COVID-19 como uma questão sociocientífica e propõe a criação de um site contendo recursos que dialogam com demandas de professores de ciências da educação básica pelo tratamento curricular deste tipo de questão. Em atividade desde 31 de março do 2020, o GT reúne participantes de nacionalidade brasileira, colombiana e portuguesa, com formação nas áreas de Biologia, Física, Química, Educação Física e Educação Ambiental. Esta diversidade, disciplinar, epistemológica e cultural, enriquece as discussões e a elaboração de propostas.

QUESTÕES SOCIOCIENTÍFICAS: O QUE SÃO E QUAL SUA IMPORTÂNCIA PARA A COMPREENSÃO DA PANDEMIA DA COVID-19?

Frequentemente nos defrontamos em nosso cotidiano com questões que, muitas vezes, envolvem debates e disputas - inclusive entre especialistas, e cujo entendimento e enfrentamento envolvem não só a contribuição de diversas áreas de conhecimento, mas a consideração de valores morais e éticos. Enquanto algumas destas questões possuem contornos geográficos específicos, como a gestão de recursos hídricos, outras, como as mudanças climáticas, têm o potencial de afetar o planeta como um todo. Enquanto algumas, como o manejo de resíduos sólidos articulam-se a questões econômicas, outras, como as terapias gênicas, trazem questionamentos éticos. A pandemia da COVID-19 é, talvez, o exemplo mais atual deste tipo de questão.

A evidente complexidade de tais questões, cujo debate envolve controvérsias, conhecimentos científicos e considerações de ordem moral e ética, tem sido explorada no campo da Educação em Ciências pelo programa de pesquisa denominado "Questões Sociocientíficas" (QSC). De acordo com Stiefel et al (2004), as QSC são questões científicas presentes no dia-a-dia e envolvem controvérsias com forte repercussão social. Para Martinez (2012), as QSC, via de regra, incluem 
dimensões ambientais (biocombustíveis, contaminação, uso da água, manipulação e disposição do lixo), de saúde pública (automedicação, anorexia e bulimia, controvérsias sobre vacinas) e éticas (experimentação com animais, terapia genética).

Os estudos sobre QSC dialogam com pressupostos, referenciais e resultados de investigações relacionados a outras subáreas da Educação em Ciências, notadamente as abordagens CTS (ciência-tecnologia-sociedade) (LIMA e MARTINS 2013) e os estudos sobre Natureza da Ciência (MARTINS, 2015). Além disso, Nurtamara et al (2018) destacam o potencial do trabalho com QSC para o desenvolvimento do pensamento crítico e de habilidades argumentativas, bem como para a compreensão de aspectos da Natureza da Ciência e dos processos de produção do conhecimento científico, por exemplo, das relações entre teoria e evidência. Finalmente, o estudo de QSC considera explicitamente as controvérsias científicas de forma a discutir dimensões históricas e sociais dos processos de produção do conhecimento científico, problematizando disputas, influências econômicas e políticas e formas de construção de consensos na ciência (REIS e GALVÃO, 2005).

Neste sentido, pode-se dizer que a agenda das QSC permite contribuições em relação a dois aspectos principais. Por um lado, explora a natureza multifacetada destas questões, que envolvem conhecimentos de diferentes áreas e demandam posicionamentos e decisões, em nível individual e/ou coletivo (EVAGOROU, 2011). Por outro, avança no que diz respeito ao desenvolvimento e à avaliação de propostas e programas curriculares (SADLER, 2011; SOUZA e GEHLEN, 2017).

Nosso entendimento da COVID-19 como uma QSC se justifica na medida que esta envolve: (i) articulações conceituais entre diferentes áreas de conhecimento e disciplinas científicas; (ii) a consideração explícita de aspectos contextuais, éticos e morais; (iii) a problematização de aspectos históricos e epistemológicos da natureza da ciência e; (iv) a análise de discursos e de informações sobre ciência que circulam em diferentes espaços midiáticos e sociais. Reconhecemos, assim, o potencial das QSC como ponto de partida para o desenvolvimento de propostas curriculares contextualizadas e interdisciplinares de questões que possuem relevância social. Entretanto, consideramos que a especialização da formação docente e a organização disciplinar do currículo podem impor dificuldades no que diz respeito à efetivação destas propostas em sala de aula.

Pensando nestes desafios inerentes ao tratamento didático de QSC e na urgência de demandas postas pelo contexto atual da pandemia da Covid19, propomos o desenvolvimento de um site que explore tais aspectos e no qual professores da educação básica possam encontrar não só esclarecimentos acerca de diferentes dimensões da pandemia mas também recursos para trabalhar conteúdos relacionados à Covid19 em suas salas de aula.

\section{CONSTRUÇÃO DE UM AMBIENTE EDUCATIVO SOBRE QSC.}

De acordo com Alves e Nascimento (2017), as novas Tecnologias Digitais de Informação e Comunicação (TDIC) desempenham importante papel no estabelecimento de novas sociabilidades e de novos ambientes educativos. Entre essas, destaca-se a criação de redes de informação e conhecimento que pode 
abranger desde blogs, chats até comunidades virtuais. Nesse sentido, Stedile e Giacomoni (2019) argumentam que o uso de tecnologias voltadas para propostas educacionais, como por exemplo um site, mostra-se como importante recurso para o processo de socialização do conhecimento.

Problematizar a COVID-19 como QSC implica identificar conhecimentos disciplinares e interdisciplinares relevantes, considerar aspectos da história e da natureza da ciência, problematizar controvérsias e combater a desinformação. $\mathrm{Na}$ elaboração do site, partimos de uma inspiração freiriana para propor perguntas que articulam a COVID-19 a suas diferentes dimensões: conceituais, históricas, econômicas, sociais, políticas, culturais, entre outras. Assim, o desenho do site, descrito no quadro 1 , foi organizado a partir da análise da pandemia com respeito a cada um destes âmbitos e do seu desdobramento em perguntas.

As atividades desenvolvidas pelos integrantes do GT incluíram: (i) buscas ativas de materiais sobre a pandemia em diferentes linguagens, suportes e contextos; (ii) atividades de leitura crítica e de análise dos discursos veiculados nestes materiais; (iii) proposição de critérios para avaliar a confiabilidade das informações sobre a pandemia que circulam nas diferentes mídias sociais; (iv) desenvolvimento de perguntas que explorem as diferentes dimensões da pandemia como uma QSC; (v) análise e proposição de estratégias didáticas; (vi) elaboração de instrumentos para avaliação da recepção do site por professores de ciências.

O site foi intitulado "Questões Sociocientíficas no Ensino de Ciências", e seu desenvolvimento implicou reuniões remotas semanais para discussão do desenho, implementação e testes. O protótipo do site foi enviado a colegas com formação em biologia, educação em ciências e tecnologia educacional, com o objetivo de validação. Uma vez finalizado foi discutido com professores da educação básica em minicursos de formação continuada. Comentários e sugestões foram incorporados ao desenho do site. 
Quadro 1: Análise da pandemia em relação a diferentes âmbitos das QSC e seus desdobramentos em perguntas motivadoras.

\begin{tabular}{|c|c|c|c|}
\hline Âmbitos das & \multicolumn{2}{|c|}{ Especificação dos âmbitos } & Perguntas motivadoras \\
\hline \multirow{6}{*}{$\begin{array}{l}\text { Interdisciplinari } \\
\text { dade } \\
\text { Conhecimentos } \\
\text { necessários ao } \\
\text { entendimento } \\
\text { da pandemia. }\end{array}$} & \multirow{6}{*}{$\begin{array}{l}\text { Que campos } \\
\text { disciplinares/ } \\
\text { acadêmicos } \\
\text { estão } \\
\text { envolvidos? }\end{array}$} & Biologia & $\begin{array}{l}\text { O que é um vírus? } \\
\text { Como se propaga? } \\
\text { Qual a importância do mapeamento do } \\
\text { genoma do vírus? }\end{array}$ \\
\hline & & Matemática & $\begin{array}{l}\text { O que é crescimento exponencial? } \\
\text { Como avaliar a } \\
\text { qualidade/confiabilidade dos dados? } \\
\text { Como entender explicações baseadas } \\
\text { em análises estatísticas de risco e } \\
\text { probabilidade? }\end{array}$ \\
\hline & & Física & $\begin{array}{l}\text { Como se criam modelos do crescimento } \\
\text { da pandemia e que permitam } \\
\text { previsões? } \\
\text { Como funciona um respirador? } \\
\text { Como radiação (ionizante) é usada na } \\
\text { esterilização de materiais hospitalares? }\end{array}$ \\
\hline & & Química & $\begin{array}{l}\text { Por que o álcool hidratado }(70 \% \mathrm{GL}) \text { é } \\
\text { mais eficiente para higienização do que } \\
\text { o álcool concentrado }(96 \% \mathrm{GL}) \text { ? }\end{array}$ \\
\hline & & Geografia & $\begin{array}{l}\text { Como a distribuição dos casos se } \\
\text { relaciona a aspectos geográficos } \\
\text { (clima/rota de contaminação)? }\end{array}$ \\
\hline & & Educação Física & $\begin{array}{l}\text { Qual a importância da atividade física } \\
\text { para a imunidade? }\end{array}$ \\
\hline \multirow{4}{*}{$\begin{array}{l}\text { Controvérsia } \\
\text { Controvérsias } \\
\text { envolvidas na } \\
\text { pandemia. }\end{array}$} & \multirow{2}{*}{$\begin{array}{l}\text { Envolvendo a } \\
\text { ciência e } \\
\text { outros } \\
\text { âmbitos }\end{array}$} & \multirow[t]{2}{*}{$\begin{array}{l}\text { Âmbitos científico, } \\
\text { sociopolíticos e } \\
\text { econômicos }\end{array}$} & $\begin{array}{l}\text { Qual a melhor solução: isolamento } \\
\text { vertical ou horizontal? }\end{array}$ \\
\hline & & & Que tipo de máscara usar? \\
\hline & \multirow[t]{2}{*}{$\begin{array}{l}\text { Internas à } \\
\text { ciência }\end{array}$} & $\begin{array}{l}\text { Construção de } \\
\text { evidências }\end{array}$ & Usar ou não usar máscaras? \\
\hline & & $\begin{array}{l}\text { Pesquisa } \\
\text { básica/clínica } \\
\text { versus urgência } \\
\text { por tratamento } \\
\end{array}$ & $\begin{array}{l}\text { A cloroquina é ou não é eficiente como } \\
\text { tratamento para a COVID-19? }\end{array}$ \\
\hline \multirow[t]{4}{*}{$\begin{array}{l}\text { Natureza da } \\
\text { Ciência } \\
\text { Segurança a } \\
\text { respeito do que } \\
\text { se sabe sobre a } \\
\text { doença e seus } \\
\text { tratamentos. }\end{array}$} & \multirow[t]{2}{*}{$\begin{array}{l}\text { Método(s) } \\
\text { científico(s) }\end{array}$} & $\begin{array}{l}\text { Inferências (e } \\
\text { ações) justificadas } \\
\text { pela pesquisa } \\
\text { experimental } \\
\text { (hipóteses, } \\
\text { experimento, } \\
\text { teste, } \\
\text { confiabilidade, } \\
\text { replicabilidade) } \\
\end{array}$ & $\begin{array}{l}\text { Por que uma vacina para a COVID-19 } \\
\text { demorará tanto tempo? } \\
\text { Como se produz um medicamento para } \\
\text { o tratamento de viroses? } \\
\text { O que é resultado falso positivo/falso } \\
\text { negativo? }\end{array}$ \\
\hline & & $\begin{array}{l}\text { Inferências (e } \\
\text { ações) baseadas } \\
\text { em observações } \\
\text { empíricas e } \\
\text { experimentos }\end{array}$ & $\begin{array}{l}\text { Sob que condições os tratamentos } \\
\text { podem ser prescritos? }\end{array}$ \\
\hline & \multirow[t]{2}{*}{ Ética } & $\begin{array}{l}\text { Propriedade } \\
\text { intelectual }\end{array}$ & $\begin{array}{l}\text { Quem detém os direitos sobre as } \\
\text { descobertas científicas? } \\
\text { Quando se justifica a quebra de } \\
\text { patentes? }\end{array}$ \\
\hline & & $\begin{array}{l}\text { Riscos, } \\
\text { consentimento }\end{array}$ & $\begin{array}{l}\text { Como se justifica a proposta de testar a } \\
\text { vacina na África? }\end{array}$ \\
\hline $\begin{array}{l}\text { História da } \\
\text { Ciência }\end{array}$ & $\begin{array}{l}\text { Casos } \\
\text { históricos }\end{array}$ & $\begin{array}{l}\text { Peste negra, } \\
\text { SARS, MERS }\end{array}$ & $\begin{array}{l}\text { O que a história nos ensina sobre as } \\
\text { pandemias? }\end{array}$ \\
\hline \multirow{3}{*}{$\begin{array}{l}\text { História das } \\
\text { ideias, práticas, } \\
\text { instituições, } \\
\text { entre outras } \\
\text { dimensões do } \\
\text { fazer científico. }\end{array}$} & $\begin{array}{l}\text { Papel do } \\
\text { cientista }\end{array}$ & Estereótipos & $\begin{array}{l}\text { Cientistas: gênios, heróis ou } \\
\text { profissionais? }\end{array}$ \\
\hline & $\begin{array}{l}\text { Papel das } \\
\text { instituições }\end{array}$ & $\begin{array}{l}\text { Universidades, } \\
\text { Institutos de } \\
\text { Pesquisa }\end{array}$ & $\begin{array}{l}\text { Como se dão as relações entre pesquisa } \\
\text { e diferentes setores da sociedade? }\end{array}$ \\
\hline & & Serendipidade/ & Existem descobertas ao acaso? \\
\hline
\end{tabular}




\begin{tabular}{|l|l|l|l|}
\hline & $\begin{array}{l}\text { Contextos do } \\
\text { trabalho } \\
\text { científico. }\end{array}$ & $\begin{array}{l}\text { Descoberta/ } \\
\text { Justificativa }\end{array}$ & $\begin{array}{l}\text { Que fatores influenciam a condução de } \\
\text { investigações e a confiabilidade nos } \\
\text { seus resultados? }\end{array}$ \\
\hline $\begin{array}{l}\text { Fake News } \\
\begin{array}{l}\text { Avaliação das } \\
\text { informações } \\
\text { que circulam } \\
\text { sobre a } \\
\text { pandemia. }\end{array}\end{array}$ & $\begin{array}{l}\text { Credibilidade } \\
\text { da fonte }\end{array}$ & Redes sociais & $\begin{array}{l}\text { Os morcegos são os transmissores do } \\
\text { SARS Cov-2? }\end{array}$ \\
\cline { 3 - 4 } & & $\begin{array}{l}\text { A vacina contra coronavírus que é } \\
\text { administrada a cães e gatos pode ser } \\
\text { usada em humanos? }\end{array}$ \\
\hline
\end{tabular}

Fonte: Os autores

\section{DESCRIÇÃO DO SITE}

O site encontra-se disponível em <www.qsc.nutes.ufrj.br>. Sua homepage inclui a apresentação da equipe e vinculação institucional, contatos e descrição de seus objetivos. Inclui uma barra de menu horizontal que leva a páginas específicas que tratam de diversas questões sociocientíficas (Figura 1).

Figura 1: Homepage do site "Questões sociocientíficas".

\section{Questões Sociocientíficas no Ensino de Ciências}

\begin{tabular}{l|l|l|l} 
Inicio & Pandemias & Mudança Climática & Gestão de Recursos Hidrícos
\end{tabular}

Frequentemente nos defrontamos em nosso cotidiano com questões que, muitas vezes, envolvem controvérsias - inclusive entre especialistas -, e cujo entendimento envolve não só a contribuição de diversas áreas de conhecimento mas a consideração de valores morais e éticos.

Como debater estas questões sóciocientificas em sala de aula? Que conhecimentos são relevantes para o debate?

Que abordagens curriculares adotar?

Neste site organizamos recursos para o tratamento destas questões sócio-científicas, a partir de uma perspectiva interdisciplinar, por meio do diálogo com pesquisas que exploram: aspectos da história da Ciência; a natureza dos processos de construção de conhecimento científico e suas relações com diferentes culturas; as relações entre ciência-tecnologia e sociedade; características do discurso científico; o desenvolvimento do pensamento crítico e de habilidades de leitura e argumentação.

Conheça e envie suas sugestões!

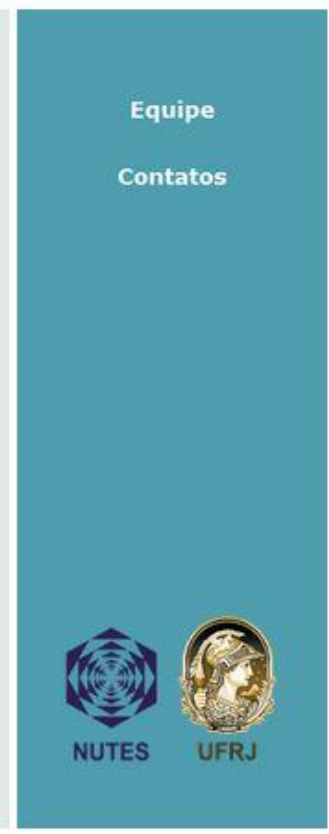

Fonte: <www.qsc.nutes.ufrj.br >.

Ao selecionar "Pandemia", o visitante é direcionado para uma página na qual encontram-se perguntas motivadoras relacionadas a cada um dos âmbitos das QSC (Figura 2). 
Figura 2: Perguntas motivadoras

\section{Questões Sociocientíficas no Ensino de Ciências

\begin{tabular}{|l|l|l|l|}
\hline Inicio & Pandemias & Mudança Climática & Gestäo de Recursos Hidricos \\
\hline
\end{tabular}

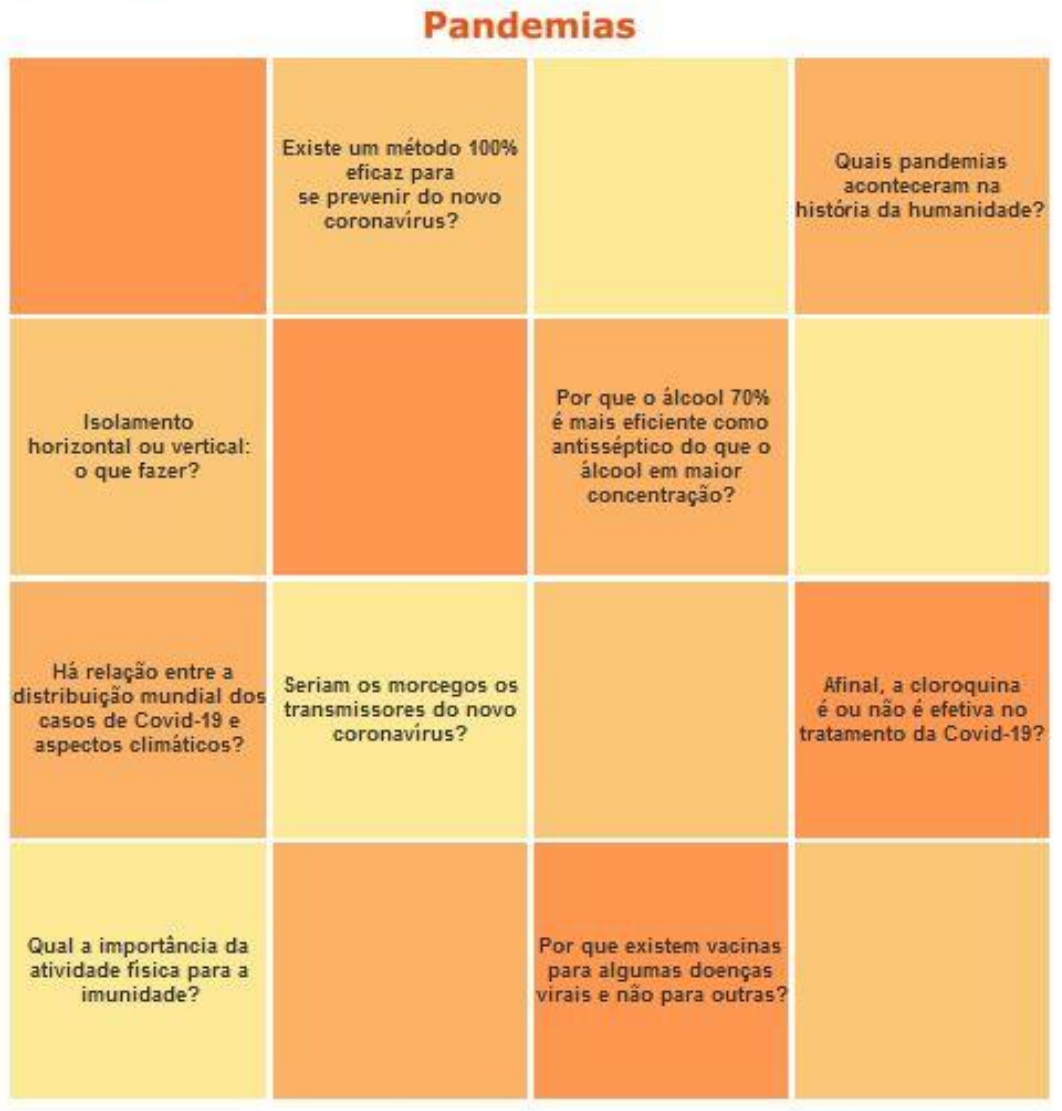

Fonte: <www.qsc.nutes.ufrj.br>.

Ao clicar em cada uma das questões, o visitante é direcionado para uma outra página (figura 3), na qual encontra uma discussão da questão e hiperlinks para artigos científicos, textos de divulgação, sites institucionais, vídeos, entrevistas, animações, que aprofundam aspectos específicos da discussão. Ao final, são apresentadas sugestões de recursos pedagógicos e de propostas de atividades em sala de aula. A opção por este formato torna o site leve e facilita o arquivamento de materiais para futura referência.

O desenho do site não inclui sugestões de atividades fechadas e valoriza a autonomia docente na adaptação e recontextualização das nossas sugestões na construção de seus planejamentos pedagógicos. 
Figura 3 - Exemplo de resposta à pergunta, hiperlinks e sugestões de recursos.

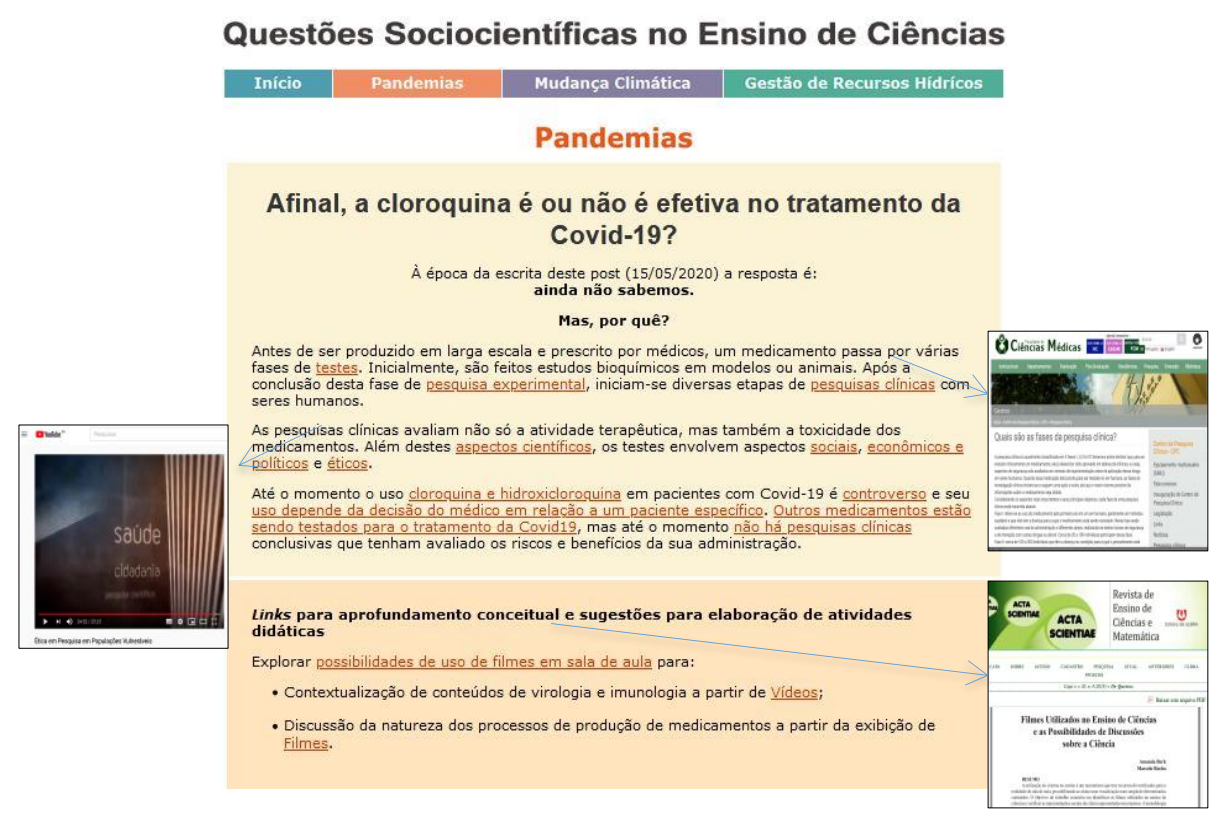

Fonte: <www.qsc.nutes.ufrj.br>.

\section{CONCLUSÕES E DESDOBRAMENTOS}

Argumentamos que a pandemia da COVID-19 é um exemplo dramático de questão sociocientífica. Controversa quanto a seu surgimento, disseminação e impactos, e possui caráter global. A velocidade do contágio, juntamente com a ausência de uma vacina e a dificuldade para estabelecer protocolos de tratamento, dificultam sua compreensão. Para além das dimensões conceituais, sua compreensão e enfrentamento demandam desde a mudança de hábitos culturais e comportamentos sociais até considerações acerca de impactos de uma possível recessão econômica em consequência dos esforços para desaceleração da taxa de contágio por meio de recomendações de isolamento.

Em tempos de distanciamento social, o desenvolvimento do site nos desafiou no que diz respeito a aspectos pedagógicos e técnicos. Destacamos o papel formativo das nossas interações e o trabalho colaborativo do grupo na definição dos conteúdos, na elaboração das perguntas, na articulação dos princípios de contextualização e interdisciplinaridade, na seleção de materiais, nas decisões sobre o desenho do site, na identificação dos materiais e na definição das estratégias e atividades sugeridas.

Atualmente está em andamento um estudo de avaliação do site com o objetivo de levantar dados sobre as percepções de professores da educação básica em relação à navegabilidade e a aspectos relacionados as atividades curriculares. As questões foram organizadas em formulário eletrônico enviado a 20 professores que têm participado de programas de formação continuada na Universidade Federal do Rio de Janeiro (UFRJ) e no Centro Federal de Educação Tecnológica Celso Suckow da Fonseca (CEFET-RJ). Resultados preliminares indicam uma recepção positiva, com destaque a facilidade de navegar pelo site e a variedade de material disponível para o uso em atividades pedagógicas. Estes resultados 
sugerem um caminho promissor na elaboração de materiais que sejam de fácil acesso, não demandem recursos tecnológicos sofisticados, mas que problematiza aspectos complexos e plurais das questões sociocientíficas e suas articulações curriculares de forma adequada. 


\title{
The COVID-19 pandemic as a socio-scientific issue: contributions from the NUTES Institute for teachers and students of Basic Education
}

\begin{abstract}
This paper deals with the COVID-19 pandemic from the perspective of socio scientific issues. It presents the development of a website aimed at addressing conceptual, historical, economic and socio-political aspects of the pandemic, in a contextualized and interdisciplinary way. It mobilized a group of schoolteachers, undergraduate and graduate students who analyzed controversies and fake news around the pandemic, defined criteria for selection of materials, and proposed motivating questions and educational activities in the production of a web page addressed to basic education science teachers. The site clarifies aspects related to the pandemic and supports curricular approaches to the theme in times of social isolation.
\end{abstract}

KEYWORDS: Socio scientific issues. COVID-19. Science education. Controversy. 


\section{AGRADECIMENTOS}

Os autores agradecem a Humberto Martins, Marcia Garcia, Rafaela Ferreira, Bruna Miceli, Jhony Coelho, Luciana Fiuza, Alberto Henrique, Samara Kister, Beatriz Modesto, Arthur Vasconcelos, Roberta Rodrigues pelas valiosas contribuições ao trabalho. Expressam também seu reconhecimento ao apoio do CNPq e da FAPERJ ao projeto.

\section{REFERÊNCIAS BIBLIOGRÁFICAS}

ALVES, J.D.N.; NASCIMENTO, M.S. A inserção e o uso de tecnologias da informação e da comunicação para a melhoria do ensino-aprendizagem: uma análise sobre a percepção do gestor de uma ETE do Recife (PE). Revista Tecnologia e Sociedade, v. 13 , n. 29 , pp. $45-64,2017$. doi: $10.3895 /$ rts.v13n29.5333

EVAGOROU, M. Discussing a Socioscientific Issue in a Primary School Classroom: The Case of Using a Technology-Supported Environment in Formal and Nonformal Settings. In SADLER T (ed). Socio-Scientific Issues in the Classroom, Dordrecht: Springer, 133-159. 2011. doi: 10.1007/978-94-007-1159-4_8

LIMA, A.; MARTINS, I. As interfaces entre a abordagem CTS e as questões sociocientíficas nas pesquisas em educação em ciências. In: Atas do IX Encontro Nacional de Pesquisa em Educação em Ciências. Águas de Lindóia-SP, 2013. Disponível em: http://www.nutes.ufrj.br/abrapec/ixenpec/atas/resumos/R01731.pdf

MARTINS, A. F. P. Natureza da Ciência no ensino de ciências: uma proposta baseada em "temas" e "questões". Caderno Brasileiro de Ensino de Física, v. 32, n. 3, p. 703-737, 2015 doi: 10.5007/2175-7941.2015v32n3p703

MARTINEZ PÉREZ, L. F. Questões Sociocientíficas na prática docente. Ideologia, autonomía e formação de professores. Editora UNESP. São Paulo- SP. 2012

NURTAMARA, L. et al. The importance socio-scientific issues of in biology learning preparing students as a 21st century society. Journal of Physics Conference Series, 1157, 2019. Doi: https://doi.org/10.1088/1742-6596/1157/2/022070

REIS, P.; GALVÃO, C. Controvérsias sócio-Científicas e Prática Pedagógica de jovens profesores. Investigações em Ensino de Ciências, Vol. 10, N.2, p.131-160. https://www.if.ufrgs.br/cref/ojs/index.php/ienci/article/view/514/311 Acesso em 10 de julho de 2020.

SADLER, T. D. Socio-scientific Issues-Based Education: What We Know About Science Education in the Context of SSI. In SADLER T (ed.) Socio-Scientific Issues in the Classroom, Dordrecht: Springer, 355-369. 2011. doi:10.1007/978-94-0071159-4_20

SOUZA, P.S.; GEHLEN, S. T. Questões Sócio-científicas no Ensino de Ciências: algumas características das pesquisas brasileiras. Ensaio Pesquisa em Educação em Ciências, v. 19, pp. 1-22, 2017. doi: 10.1590/1983-21172017190109. 
STEDELI, N.L.R; GIACOMONI, R. R. F. Construção e avaliação de um museu virtual de resíduos sólidos. Revista Brasileira de Educação Ambiental. v.14, n.3, pp. 206219, 2019. doi: 10.34024/revbea.2019.v14.9410

STIEFEL, B. et al. (2004). Impacto de um Programa de Formação com uma Orientação CTS/PC nas Concepções e Práticas de Professores. In: MARTINS, I. P.; PAIXÃO, F.; VIEIRA, R. M. (Orgs.). Perspectivas Ciência-Tecnologia-Sociedade na Inovação da Educação em Ciência. Aveiro: Universidade de Aveiro, p. 89-97, 2004.

Recebido: 08/05/2020

Aprovado: 01/08/2020

DOI: $10.3895 /$ rts.v16n44.12236

Como citar: MARTINS, I. G. R.; et.al. A pandemia da COVID-19 como questão sociocientífica: aportes do Instituto NUTES para professores e estudantes da Educação Básica. R. Tecnol. Soc., Curitiba, v. 16, n. 44, p. 31-42, ed. esp. 2020. Disponível em: https://periodicos.utfpr.edu.br/rts/article/view/12236. Acesso em: XXX.

Correspondência:

Direito autoral: Este artigo está licenciado sob os termos da Licença Creative Commons-Atribuição 4.0 Internacional. 